\title{
Host receptor tyrosine kinases mediate HCV entry and are a promising new target for antiviral therapy
}

Epidermal growth factor receptor (EGFR) and ephrin receptor A2 (EphA2) have a key role in $\mathrm{HCV}$ entry, and inhibition of these host receptor tyrosine kinases (RTKs) could provide a viable new avenue for antiviral therapy.

\section{Clinically licensed PKIs ...} developed for cancer treatment, have significant antiviral

\section{activity 77}

Thomas Baumert and colleagues used a functional high-throughput RNA interference screen to identify host factors necessary for HCV entry, because HCV entry is required for viral infection to be initiated, disseminated and maintained. They chose to focus their efforts on cellular kinases because their importance in key cellular processes has been established and there are clinically approved protein kinase inhibitors (PKIs) already available for the treatment of cancer.

EGFR and EphA2 were identified by the screen, and silencing of their individual messenger RNAs confirmed their role as cofactors for HCV entry. By inhibiting EGFR and EphA2 with existing PKIs, the researchers showed that $\mathrm{HCV}$ infection by all major HCV genotypes and viral escape mutants was impaired both in cell culture and in an in vivo human liver chimeric mouse model. The mechanism by which EGFR and EphA2 mediate HCV entry was found to be via regulation of CD81-claudin-1 coreceptor associations and viral glycoprotein-dependent membrane fusion.

"Clinically licensed PKIs such as erlotinib and dasatinib, which were developed for cancer treatment, have significant antiviral activity," explains Baumert. "These results suggest that small molecules or antibodies targeting host cell kinases as HCV entry factors may offer a perspective for urgently needed antiviral strategies for prevention of HCV infection and treatment of resistance."

The interactions between the signaling pathways involved in regulating $\mathrm{HCV}$ entry and the kinase network identified by Baumert and colleagues are now being characterized. In addition, nextgeneration PKIs and novel monoclonal anti-RTK antibodies that inhibit viral entry are being screened in preclinical models. There is also a plan to assess the toxicity and antiviral effect of erlotinib (which is an EGFR inhibitor) in a randomized controlled trial in patients chronically infected with HCV.

Natalie J. Wood

Original article Lupberger, J. et al. EGFR and EphA2 are host factors for hepatitis $\mathrm{C}$ virus entry and possible targets for antiviral therapy. Nat. Med. doi:10.1038/nm.2341 